Images du travail, travail des images

1 | 2016

Quand les groupes professionnels se mettent en images

\title{
L'image, miroir du compagnonnage au XIXe siècle
}

The figurative dialect of professional groups

\section{Laurent Bastard}

\section{CpenEdition}

\section{Journals}

Édition électronique

URL : http://journals.openedition.org/itti/1285

DOI : 10.4000/itti. 1285

\section{Éditeur}

Université de Poitiers

\section{Référence électronique}

Laurent Bastard, «L'image, miroir du compagnonnage au XIXe siècle », Images du travail, travail des images [En ligne], 1 | 2016, mis en ligne le 01 février 2016, consulté le 14 avril 2021. URL : http:// journals.openedition.org/itti/1285; DOI : https://doi.org/10.4000/itti.1285

Ce document a été généré automatiquement le 14 avril 2021.

Images du travail, travail des images 


\section{L'image, miroir du compagnonnage au XIXe siècle}

The figurative dialect of professional groups

Laurent Bastard

1 Le Compagnonnage est constitué d'un ensemble d'associations (les compagnons disaient encore au milieu du XXe siècle des sociétés) destinées à assurer un perfectionnement professionnel et moral à de jeunes ouvriers d'une trentaine de métiers différents. Ces sociétés sont des fraternités de type initiatique. Elles pourvoient à l'embauche de leurs membres durant leur tour de France et leur assurent secours et assistance. Ces associations émergent historiquement au XVe siècle et ont perduré jusqu'à nos jours. Leur permanence n'est pas allée sans adaptations et transformations et le XIXe siècle est celui durant lequel elles ont été confrontées à de profondes tensions face à un environnement social, politique et technique en pleine mutation. C'est aussi à cette époque que les compagnons découvrent la puissance de l'image comme vecteur de nouvelles idées.

\section{Auteurs, techniques et diffusion}

2 L'iconographie compagnonnique du XIXe siècle est constituée de dessins aquarellés puis de lithographies. Les uns et les autres présentent des spécificités selon les corps de métier dont ils relèvent, mais ont connu une importante diffusion. Cependant, malgré leur présence dans les sièges compagnonniques et les musées, chez les collectionneurs privés et dans les salles des ventes, ces images n'ont guère intéressé les historiographes du Compagnonnage.

\subsection{Une source négligée}

3 Tout objet d'étude historique suppose des matériaux. Ils ne manquent pas en ce qui concerne le Compagnonnage, qu'il s'agisse d'objets et d'écrits, provenant des 
compagnons eux-mêmes (archives et Mémoires), issus des institutions de police ou de justice ou encore de la presse.

4 A ces catégories s'ajoutent les images, nombreuses puisqu'elles peuvent prendre la forme de dessins gouachés ou aquarellés, de gravures, de lithographies, de peintures sur toile ou bois et enfin de photographies.

Illustration 1. Dauphiné la fidélité compagnon passant charpentier bondrille pour la vie.

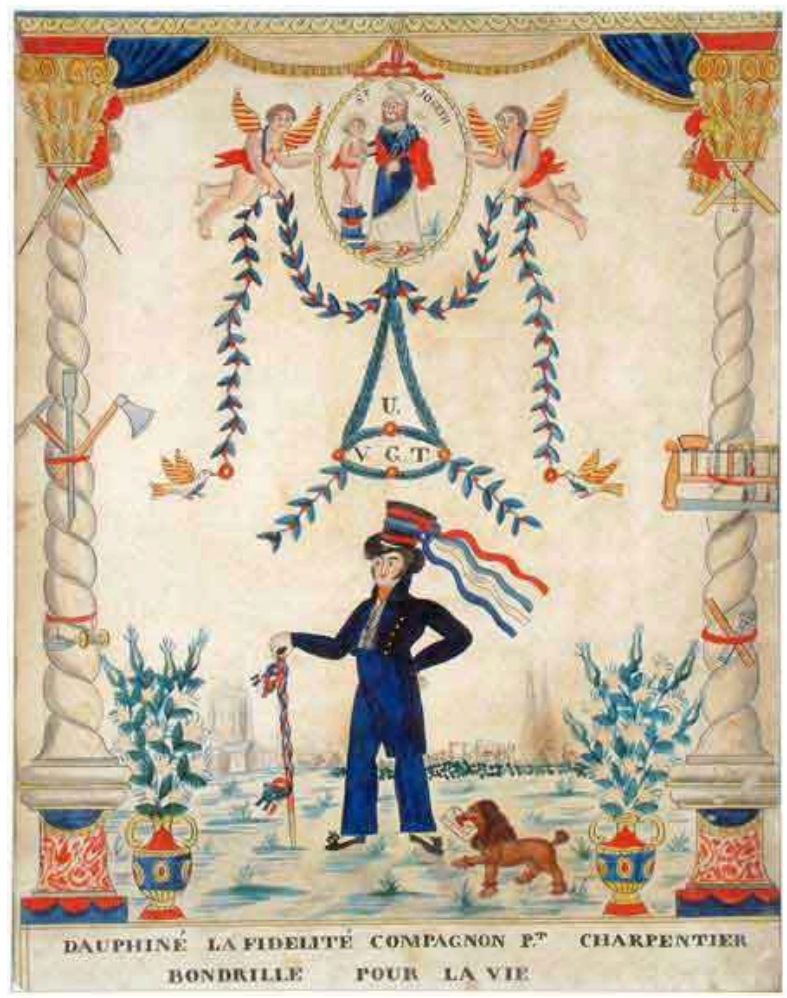

Dessin aquarellé, 47 × 37 cm, Etienne LECLAIR, Bordeaux, c. 1820-1830.

5 L'iconographie est importante, tant sur le plan de la quantité que du contenu. Pourtant, cette source d'information précieuse sur le Compagnonnage au XIXe siècle est demeurée assez négligée des historiens. Lorsqu'en 1901 Etienne MARTIN SAINT-LÉON publie Le Compagnonnage, son histoire, ses coutumes, ses règlements et ses rites, il signale, sans préciser le lieu visité, qu' "au mur sont accrochées quelques lithographies : le roi Salomon représenté dans un costume qui rappelle assez celui des monarques peints sur les cartes à jouer ou encore la Réconciliation des compagnons, gravure tirée d'une œuvre dramatique de Perdiguier mais qui évoque aussi le souvenir de la manifestation de 1848. Nous avons vu également quelques gravures plus anciennes et plus curieuses représentant divers épisodes de l'initiation des compagnons. $»^{1}$. L'historien ne s'attarde pas sur ces images qu'il juge ou trop naïves ou trop hermétiques. 


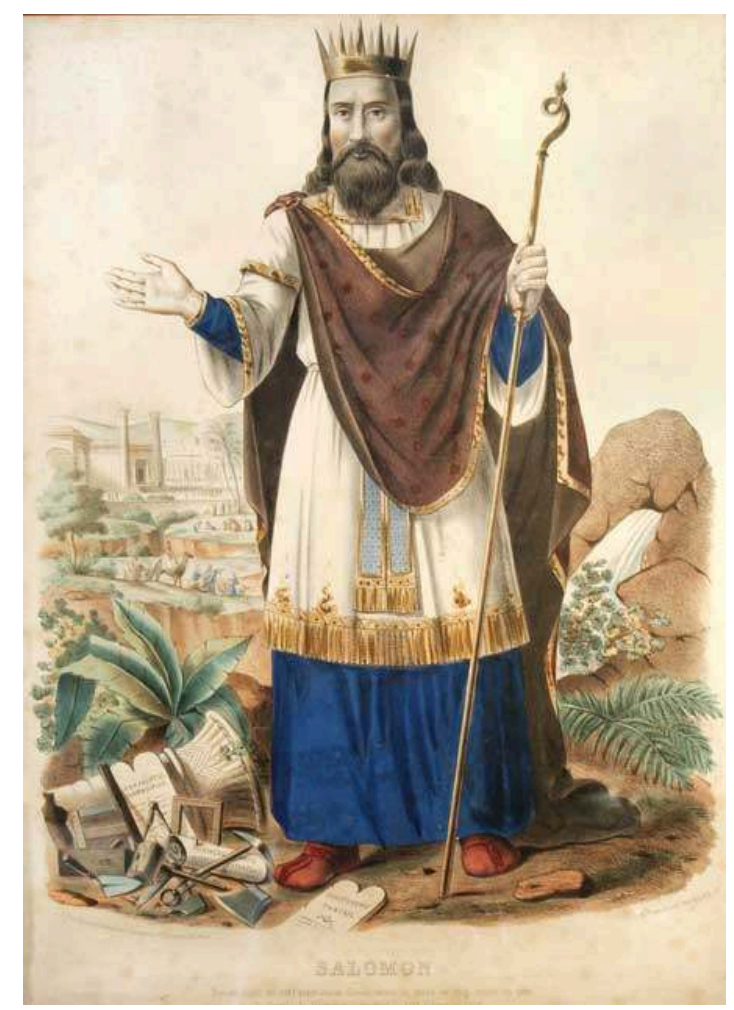

Lithographie, 53 x 39 cm, Agricol PERDIGUIER dit Avignonnais la Vertu, compagnon menuisier du Devoir de Liberté, et BECQUET, imprimeur-lithographe, Paris, 1862.

En 1966, Émile COORNAERT2 ${ }^{2}$, étudie un peu plus les images, commentant celles qui illustrent son livre : les trois fondateurs, par Perdiguier, le Souvenir du tour de France du vitrier Robert, une conduite de Leroux ou le brevet de compagnon initié des charpentiers du Devoir de Liberté. Incidemment, à la fin du chapitre V sur « La vie des compagnons ", il écrit que « Dans la grisaille quotidienne où se déroulait l'existence du commun, les compagnonnages inséraient un tableau d'une couleur intense, d'un reflet digne d'images d'Épinal. De fait, des artistes dont le plus connu est Leclair, ont multiplié les estampes de leurs cérémonies. Leur fraîcheur et leur mouvement raniment une vie particulière, constamment inspirée de traditions, réglée par des fidélités inébranlées. "

D'autres auteurs, dans diverses publications, ont commenté ici et là un dessin ou une lithographie, mais aucune étude spécifique n'a vu le jour à l'exception de celle que Roger LECOTTÉ publia en 1948 dans Artisans et paysans de France ${ }^{3}$.

En 1995, j'ai pour ma part consacré une étude à «L'iconographie compagnonnique du XIXe siècle» dans le catalogue de l'exposition «Le Compagnonnage, chemin de l'excellence » qui fut présentée au musée national des arts et traditions populaires. Puis en 2005 a été publiée dans le volume 7 des Fragments d'histoire du Compagnonnage édité par le musée du Compagnonnage de Tours, la conférence de Jean PHILIPPON sur "L'imagerie compagnonnique ou le pouvoir évocateur de l'image ». Enfin, en 2010, j'ai analysé une vingtaine de dessins et de lithographies sous le titre Images des compagnons du Tour de France. ${ }^{4}$ 


\subsection{Une vaste production}

9 La diversité de ces images (dessins et lithographies) n'a pas été la même selon les corps de métiers. Les charpentiers et les couvreurs du Devoir sont sans doute ceux qui ont fait peindre le plus de portraits en pied et de conduites (cortèges) par Leclair et Lemoine, puis éditer de lithographies. Les uns et les autres peuvent paraître stéréotypés alors qu'il n'en est rien, car les peintres personnalisaient leurs dessins aquarellés et les dessinateurs recomposaient les lithographies au fil des lieux et des époques. Une cinquantaine au moins d'images différentes sont connues chez les charpentiers et les couvreurs.

La diversité iconographique est légèrement inférieure chez les charrons, les tonneliersdoleurs, les maréchaux, les cordonniers, les charpentiers du Devoir de Liberté. Elle l'est encore plus chez les menuisiers, les sabotiers, les tisseurs-ferrandiniers, les toiliers, les bourreliers, les tailleurs de pierre du Devoir, ainsi que chez les tonneliers-foudriers du Devoir de Liberté. Les autres corporations n'ont fait peindre ou éditer qu'une ou deux œuvres connues aujourd'hui. Certaines ne semblent avoir rien produit.

11 Ce constat ne tient pas compte des quelques en-têtes connus de correspondances et de règlements dessinés ou imprimés, ni, bien sûr, de découvertes futures. Si l'on peut comprendre que de petits corps sur le déclin et peu structurés aient renoncé, faute de ressources, à faire imprimer des lithographies au nom de leur société, on s'interroge sur l'absence de dessins aquarellés au nom d'un compagnon. De même, pourquoi les tanneurs, les tailleurs de pierre, les serruriers et les menuisiers, qui étaient encore forts de plusieurs centaines de membres au milieu du XIXe siècle, sont-ils demeurés si discrets? Leurs règlements ne défendaient pas l'édition d'estampes, aussi est-il permis de se demander si cet effacement ne résulte pas d'un état d'esprit particulier, celui propre aux corps qui sont assurés de leur ancienneté et de leur légitimité et qui n'éprouvent aucun besoin de démontrer aux autres par l'image qu'ils constituent d'authentiques et vénérables compagnonnages ${ }^{5}$. Mais alors, pourquoi les charpentiers, corporation légitime et ancienne s'il en est, ont-ils produit une iconographie aussi abondante ? Peut-être, tout simplement, a-t-il manqué aux précédents un compagnon qui ouvre la marche, car si les charpentiers, après l'abondante production du peintre bordelais Leclair sous la Restauration, ont continué, cette fois par la lithographie, à produire des images, sans doute était-ce pour perpétuer une «tradition » bien ancrée chez eux. L'effet d'imitation, le suivi de leurs prédécesseurs, sont des attitudes maintes fois constatées chez les compagnons ${ }^{6}$.

12 Si la diversité des images est plus ou moins importante selon les corps de métier, les quantités produites ont globalement été notables au cours du XIXe siècle, compte tenu du nombre d'exemplaires conservés dans les musées, dans les sièges des compagnons, chez des particuliers ou signalés lors des ventes d'antiquités.

13 Si les dessins de Leclair et de Lemoine, ou d'autres peintres, demeurent assez rares puisqu'il s'agit de pièces uniques, les lithographies ont été éditées en de nombreux exemplaires, sans que l'on puisse malheureusement connaître le volume des tirages pour chacune d'elles. Cette production en série a permis de satisfaire une demande autant que de la susciter. 


\subsection{Qui dessinait ou éditait ces images?}

Il pouvait s'agir de non-compagnons, tel Etienne Leclair, piqueur à Bordeaux (employé du service de voirie municipale), qui peignit une trentaine de portraits-souvenirs et de conduites $^{7}$ (recensés, il y en eut évidemment bien davantage), pour des compagnons de passage, entre 1818 et 1830 environ. Ou d'Auguste Lemoine, peintre qui résidait à Angers et qui en réalisa une quinzaine dans les années 1830-1840, surtout pour les couvreurs et les charpentiers mais aussi pour les charrons.

Illustration 3. Champ de conduite des compagnons passants couvreurs bondrilles pour la vie de Tours.

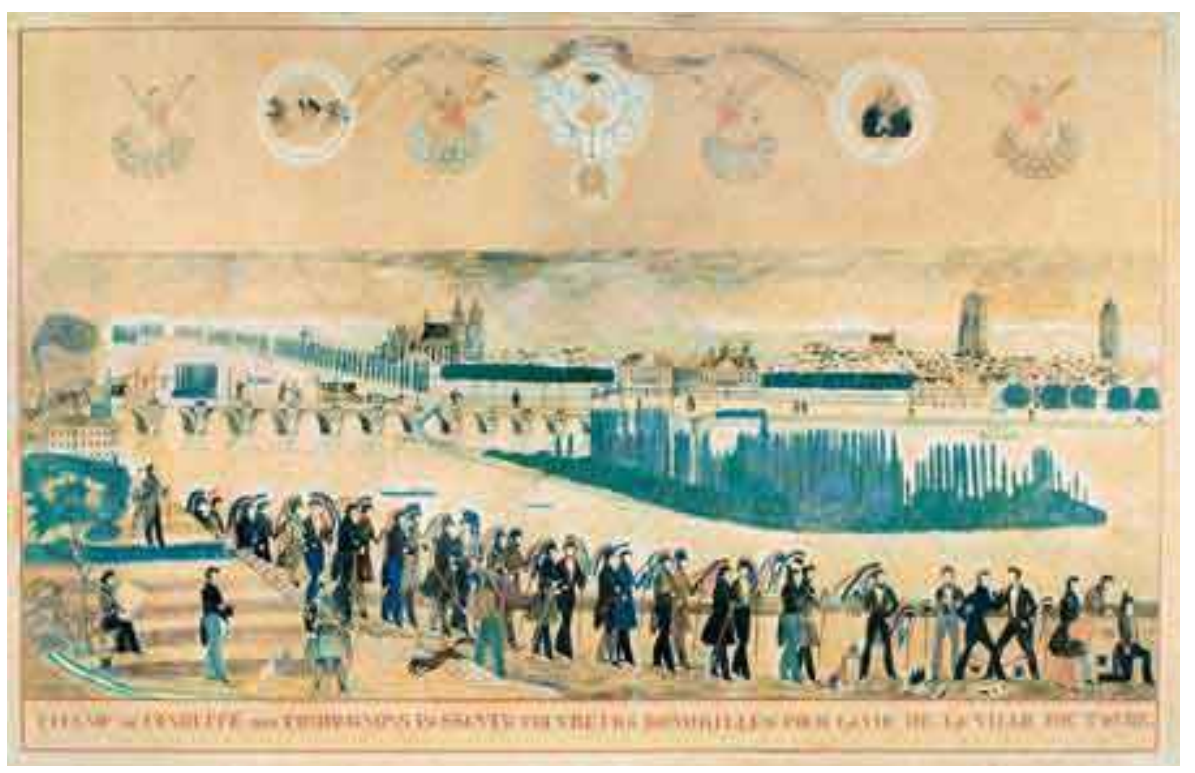

Dessin aquarellé, 64,5 x 100 cm, Auguste LEMOINE, Tours ; 1838.

D'autres étaient des compagnons doués d'un bon "coup de crayon». Le couvreur Besnard dit Langevin la Fidélité en réalisa au moins deux en 1848 pour Moreau dit Langevin la Couronne et Rousseau dit Tourangeau la Sagesse. Chez les doleurs ${ }^{8}$, on peut citer les tourangeaux Auguste et Alexis Mazery, auteurs en 1847 d'un beau règlement dessiné et rehaussé d'aquarelle. Un charron, Auguste Petitpas, dit Beauceron le Bien aimé, réalisa pour sa part un beau souvenir en 1859 pour Guépin l'Ille d'amour, où voisinent le compagnon en pied, sainte Catherine, une « guilbrette» (rite de reconnaissance), une conduite et autres figures emblématiques. On pourrait multiplier les exemples jusqu'à la fin du XIXe siècle et même au-delà, car ce type d'images n'a jamais été totalement supplanté par la lithographie.

En ce qui concerne cette seconde catégorie ${ }^{9}$, il faut distinguer trois partenaires. Il y a d'abord le compagnon qui fait éditer les estampes, puis le dessinateur qui exécute l'œuvre et enfin l'imprimeur qui les fait tirer. On peut supposer que dans la majorité des cas, le dessinateur était l'employé de l'imprimeur. Mais quelle était la part de création du compagnon ? Établissait-il un croquis, un dessin plus ou moins abouti qui servait de modèle ou donnait-il seulement des indications verbales, à charge pour le dessinateur de mettre tout cela en forme? Le premier fournissait-il au second des images antérieures, compagnonniques ou non, en laissant à l'artiste le soin de s'en inspirer pour composer une œuvre nouvelle? Ce dernier puisait-il dans la 
documentation de l'imprimerie? Aucune de ces questions n'a trouvé de réponse car il n'a pas été découvert de contrat ou de courrier détaillant la part prise par le compagnon et le dessinateur dans l'élaboration de l'œuvre.

Illustration 4. Souvenir des compagnons passants charpentiers

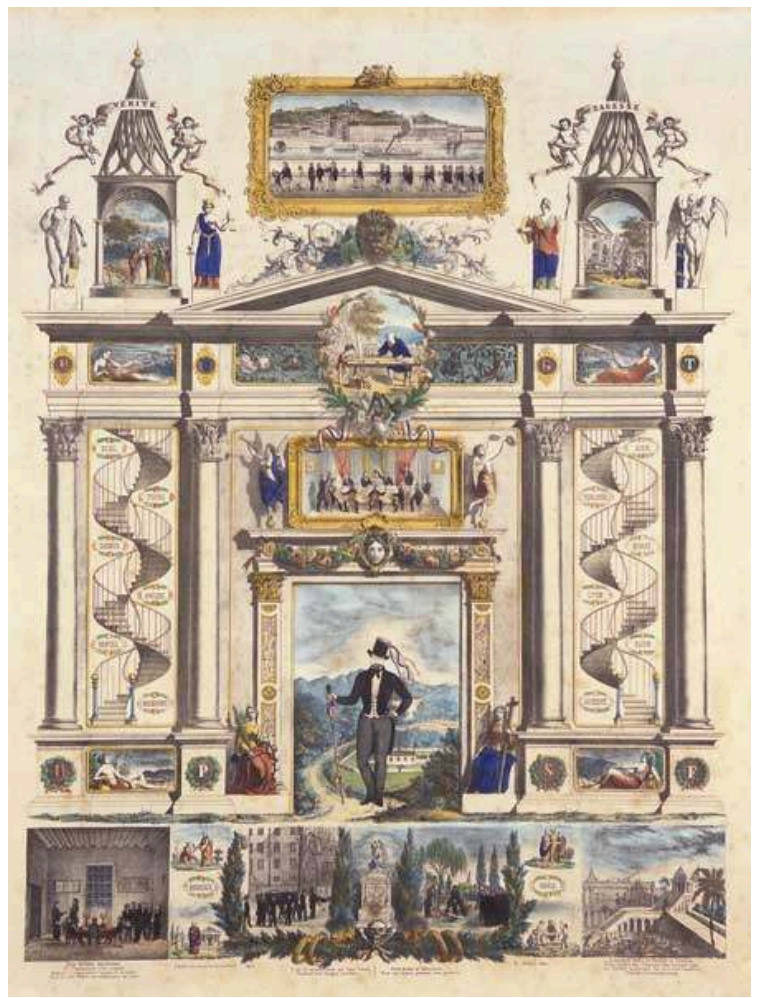

Lithographie, 85 x 62 cm, auteurs : Mathelin et Maillet, à Lyon, c. 1850-1860.

Quoi qu'il en soit, les compagnons et les sociétés qui firent éditer des lithographies se comptent par dizaines et leur recensement demeure à établir. Certains en firent réaliser une seule, d'autres plusieurs sur des thèmes différents. Du nombre sont Agricol Perdiguier dit Avignonnais la Vertu, compagnon menuisier du Devoir de Liberté ${ }^{10}$, et Jean-Baptiste Bourguet dit Forézien Bon Désir, compagnon tisseur-ferrandinier du Devoir, respectivement éditeurs de huit et de dix lithographies différentes.

Ce procédé d'impression présentait l'avantage sur le dessin aquarellé de pouvoir rapidement satisfaire la demande d'un compagnon qui souhaitait disposer d'un souvenir de son tour de France ou de diffuser largement des idées nouvelles. En quoi consiste-t-il?

\subsection{La lithographie : technique et diffusion}

Il s'agit d'une technique d'impression découverte vers 1795 par l'autrichien Aloys Senefelder. Elle nécessite l'emploi d'une pierre calcaire à grain très fin d'où son nom (du grec lithos, pierre et graphein, dessiner). Après lui avoir fait subir une préparation particulière, l'artiste y réalise son dessin avec un crayon lithographique, une plume ou un pinceau, trempé dans l'encre lithographique, épaisse et grasse. Puis l'impression s'opère en humidifiant la pierre et en passant sur elle un rouleau de cuir imprégné d'encre grasse. Celle-là repousse l'eau sur toutes les parties où ne figure pas de dessin 
pour se concentrer sur celui-ci. La feuille de papier, appliquée sur la pierre, est alors soumise à l'action de la presse à bras et se trouve imprimée.

Elle peut demeurer telle quelle, en noir, ou bien être coloriée. Des ouvriers de l'imprimerie mais plus souvent des personnes travaillant à domicile, des femmes surtout, appliquaient des couleurs au pinceau sur tout ou partie de l'estampe. C'était un travail délicat, qui devait respecter les consignes de l'éditeur. Souvent, seuls les rubans des compagnons représentés, justement parce qu'on les appelle des couleurs, étaient peints en rouge, bleu, jaune, vert, etc. selon les sociétés concernées. Il est d'ailleurs à noter qu'à la même époque, lorsque les compagnons prennent l'habitude de se faire photographier après leur réception, au format carte de visite, ils colorient souvent à la gouache leurs couleurs, puisqu'on ne connaissait encore que les tirages en noir et blanc ou en sépia.

21 Il n'y a pas de ville plus spécialisée qu'une autre pour l'impression des lithographies compagnonniques. Elles ont été réalisées à Paris, Orléans, Tours, Saumur, Nantes, La Rochelle, Bordeaux, Toulouse, Saint-Etienne, Lyon... Les imprimeurs ne paraissent pas avoir établi de liens exclusifs avec les compagnons, car beaucoup d'entre eux sont connus pour avoir produit toutes sortes d'estampes profanes, des images pieuses et des illustrations de livres. Certains étaient assez renommés, comme Lemercier, établi à Paris, rue de Seine, qui imprima les œuvres de Manet et d'Odilon Redon mais aussi une conduite des compagnons maréchaux du Devoir. Il en est de même des imprimeries Clarey-Martineau et Juliot, à Tours ou Charpentier, à Nantes.

Une fois imprimées, ces lithographies constituaient un stock que le compagnon éditeur, ou une société compagnonnique, se devaient d'écouler. Le compagnon en avisait par courrier tous ceux qu'il connaissait, ou les sièges des associations locales et y plaçait ses estampes en dépôt. Perdiguier faisait ainsi et demandait à ceux qui se déplaçaient d'emporter avec eux les prospectus de ses lithographies. À partir de 1880, lorsque se met en place la presse compagnonnique, quelques compagnons saisissent la balle au bond pour insérer des annonces ou même de petits articles mi-informatifs, mipublicitaires sur leur dernière œuvre ${ }^{11}$.

Dans tous les cas, qu'il s'agisse de dessins coloriés ou de lithographies, le bouche à oreille devait jouer un rôle essentiel, car l'information était propagée de ville en ville par les compagnons qui accomplissaient leur tour de France. Celui qui avait acheté un beau souvenir ici devait s'empresser de le faire admirer par ses frères dès la ville suivante, lesquels s'empressaient d'en acquérir un à leur tour.

Les compagnons éditeurs d'estampes devaient tirer quelque profit de l'opération. On manque de données sur les coûts d'impression autant que sur les prix de vente, qui devaient différer selon les villes et la réputation des imprimeurs. On notera qu'à Tours, en janvier 1865, cinq «tableaux de compagnonnage ", achetés quelques mois plus tôt, étaient estimés cinquante francs, mais ils étaient encadrés de bois de noyer. Vers 1880, Bourguet proposait son estampe sur le Voyage de la Sainte-Baume à 1,25 franc l'exemplaire. En 1891, le cordonnier Fardin vendait son Temple compagnonnique 2,50 à 3 francs selon le papier, et Charue vendait 3 francs le Génie du Compagnonnage. Il s'agissait de grands formats. A la même époque, le salaire journalier d'un ouvrier charpentier parisien était de 8 francs; il dépensait chaque jour 2,25 francs pour ses repas au marchand de vin (petit déjeuner, repas du midi et collation de l'après-midi), auxquels s'ajoutait celui du soir à son domicile. Sans nous avancer plus avant, il nous semble 
qu'une estampe représentait un coût non négligeable pour un ouvrier dont l'essentiel du salaire était absorbé par l'alimentation, le loyer et l'habillement.

\section{Fonctions et contenu}

$\mathrm{Au}$ début du XIXe siècle les images des compagnons ne paraissent avoir servi que de souvenir à l'issue du tour de France. Ce sont les instantanés d'une vie compagnonnique qui ne dure que quelques années, le temps du séjour des compagnons dans les villessièges du tour. Progressivement, la vie des compagnons se poursuit au-delà de son retour dans leurs foyers et les images s'enrichissent de notions symboliques et philosophiques issues de la franc-maçonnerie à laquelle certains d'entre eux adhèrent. Les images passent alors du statut de souvenirs individuels à celui de support d'un enseignement crypté, diffusé non seulement auprès des compagnons acquéreurs mais aussi exposé en permanence au sein de leurs lieux de réunion, chez la Mère.

\subsection{Pourquoi ces images?}

Il faut distinguer deux catégories d'images selon leur destination. La première comprend les images personnalisées, destinées à raviver le souvenir des bons moments du tour de France chez le compagnon qui l'acquiert. Il l'exposera chez lui, une fois son tour achevé ou après qu'il ait « remercié » (quitté) sa société. Ce type d'image peut être un dessin aquarellé ou une lithographie représentant le compagnon en pied ou bien lors d'une conduite. La seconde catégorie est constituée de lithographies qui ont une vocation pédagogique. Il s'agit pour ceux qui les ont composées d'instruire leurs frères, de propager des idées, de les appeler à partager un but ou un idéal. Les acquéreurs adhèrent aux idées véhiculées par les images ou bien sont séduits par leur caractère ésotérique qui renforce leur sentiment d'appartenir à une société à mystères et à une élite d'initiés. Ce type d'image se développe autour des années 1840 pour culminer à la fin du XIXe siècle.

Toutes ces images ont eu aussi une fonction d'ornement des sièges compagnonniques. Quelques photos d'intérieur de chambres ou de salles à manger chez les Mères, prises dès la fin du XIXe siècle montrent des murs décorés d'estampes encadrées, comme l'a remarqué Martin Saint-Léon. Elles ne servent pas seulement qu'à embellir un lieu, elles le "compagnonnisent ", lui confèrent une atmosphère qui le différencie d'une salle d'auberge profane. Plus encore, elles sont là pour susciter le respect et les questions des aspirants comme pour rappeler leurs devoirs aux compagnons. C'est ce qu'a bien exprimé le compagnon plâtrier Joseph Potier dit Le Bien-Aimé de Saint-Georges de Reintembault dans son poème sur " Le réfectoire des CC $\therefore$ du Devoir » :

«IV. On voit sur la paroi du mur / De beaux cadres, des panoplies, / Des attributs sur clair-obscur / Et des gravures fort jolies; L'une d'un plâtrier actif, / Par la Sincérité signée, / De Vil-neuv-de-Berg est natif; C'est une œuvre vraiment soignée ${ }^{12}$.

V. De ce tableau très réussi / Un voile cache les mystères, / C'est le plus grand qui jusqu'ici / Donne les plus beaux caractères; / Chaque chose nous montre un but / Où l'on peut lire une devise, / C'est un poème, un attribut / Des enfants du Père Soubise.

VI. Dans deux grands cadres, les portraits / Des fondateurs, Jacques, Soubise ; / Tout 
près, un autre, plein d'attraits, / Montre Magdeleine soumise, / La chapelle de Saint-Pilon, / La montagne de Sainte-Baume; / Les voyageurs en ce vallon / trouvent à leurs maux un doux baume. $»^{13}$

\subsection{Permanence et évolution des contenus} colorié. Jusqu'à la fin du XIXe siècle, les images perpétuent le thème du "souvenir ", avec le compagnon en pied devant un paysage urbain ou par celui de la Sainte-Baume, ou bien encore la conduite. De même, la présentation des outils ainsi que les angelots continuent à figurer sur les images. Mais on constate que les deux thèmes principaux du compagnon en pied et de la conduite ne sont plus les seuls. Ils n'occupent plus la totalité de l'image, à quelques exceptions près, mais se trouvent de plus en plus traités en vignettes de petites dimensions et associés à d'autres figures, symboles, scènes, mythes, etc. La composition devient chargée, comme s'il s'agissait de « tout dire » en un espace réduit. En d'autres termes, on est passé en un demi-siècle du simple au compliqué et ce constat correspond exactement à ce qui s'est produit à la même époque au niveau des rituels de réception et des « livres de Devoir $»^{14}$.

\subsection{Se singulariser pour exister}

31 Ce phénomène s'explique par la position du Compagnonnage dans la société du XIXe siècle. Les Devoirs, après la Révolution, ont perdu leurs repères traditionnels : le roi, la religion chrétienne, les maîtres des corporations. La loi Le Chapelier a supprimé « toute espèce de corporation " d'ouvriers comme de maîtres, consacrant le principe de la liberté individuelle. Dans le même temps le monde ouvrier se déchristianise et radicalise ses positions envers ce qui va s'appeler bientôt le patronat. La notion de classe ouvrière se répand, à laquelle les Devoirs, sélectifs par nature, ne peuvent souscrire. L'opposition ouvriers-patronat entendue comme une réalité permanente des sociétés capitalistes, peine à être admise par les compagnons. Même ceux qui demeurent offensifs, comme les tisseurs, les charpentiers et les maréchaux, ne cessent le travail que pour des motifs déterminés et souvent localement. Leur démarche ne s'inscrit pas dans une stratégie globale, d'autant que les sociétés deviennent de plus en plus, au fil des ans, des mouvements mixtes, associant fraternellement patrons et 
ouvriers, tous compagnons, et que l'ambition de la plupart de ceux qui sont salariés est de fonder un jour ou l'autre leur entreprise.

Dans ce contexte tendu, les compagnonnages n'ont que le choix de se fondre dans la masse et de rejoindre la société de l'Union des travailleurs du tour de France, les sociétés de secours mutuels ou les chambres syndicales ouvrières. Une minorité l'a fait. Ou bien il leur reste à affirmer encore plus leurs différences vis-à-vis des autres ouvriers.

Mais comment se singulariser alors que les vieilles formes rituelles et iconographiques héritées de l'Ancien Régime ne sont plus toujours comprises par les compagnons? Ce ne sera pas la simplicité qui parle au grand nombre qui va être privilégiée, mais la complexité et même un certain ésotérisme. Il faut désormais s'affirmer comme membres d'une société d'initiés, d'élus distincts du "vulgaire», comme les affiliés à une aristocratie ouvrière, un groupe de professionnels d'élite, tant sur le plan du métier que de la morale. Le prestige attaché au titre de compagnon se pare de mystère.

En complexifiant leur Devoir, les compagnons du XIXe siècle n'ont pas seulement cherché à exprimer les mêmes valeurs, la même morale, sous d'autres formes plus contemporaines. Ils y ont aussi, souvent, intégré de nouveaux concepts, développé des légendes, échafaudé des généalogies, imaginé des mythes fondateurs, toutes choses inconnues un demi-siècle auparavant.

\subsection{L'importance des emprunts maçonniques}

35 Où les compagnons ont-ils pris la plupart des matériaux de leur nouveau langage? Logiquement, dans ce qui leur paraissait être une institution proche de la leur par sa structure initiatique, sa recherche de fraternité, son lien avec le métier (symbolique, mais lien tout de même), son ancienneté qui la légitimait, son prestige qui était celui d'un groupe d'hommes capable de réunir le modeste travailleur et le prince de sang. Il s'agit de la Franc-maçonnerie. Elle a servi de source aux compagnonnages en recherche d'identité.

Comment expliquer que ces deux institutions se soient rapprochées alors qu'avant la Révolution elles n'ont guère entretenu de relations? Entendons-nous d'abord sur les mots. Pour ce qui nous occupe, le Compagnonnage, comme la Franc-maçonnerie, ce ne sont point des institutions, mais d'abord des hommes. C'est méconnaître que tout se fait par eux seuls que d'évoquer la "rencontre du Compagnonnage et de la Francmaçonnerie au XIXe siècle", "l'influence de la Franc-maçonnerie sur le Compagnonnage", voire "l'emprise de la Franc-maçonnerie», comme l'ont fait certains historiens et surtout des compagnons dans leurs journaux au cours de la seconde moitié du XXe siècle ${ }^{15}$. Par commodité et par raccourci, on peut être amené à user de ces formules, mais elles ne doivent pas faire oublier que, désignées par des mots au singulier (la Franc-maçonnerie, le Compagnonnage), elles sont trop générales et ne prennent pas en compte toutes ces multiples relations individuelles tissées dans le cadre des relations sociales. De plus, elles négligent la grande diversité des Devoirs, des métiers compagnonnisés, des rites, des sociétés, des compagnons, comme des obédiences, des loges et des hommes qui les composent.

Or, que s'est-il passé au cours du XIXe siècle ? D'une part, le niveau d'instruction des compagnons, comme celui du monde ouvrier, s'est constamment élevé par l'alphabétisation et la lecture. Une fois achevés leurs trois années ou plus de tour de 
France, beaucoup d'entre eux, et de plus en plus au cours du siècle, ont conservé des liens étroits avec l'activité des jeunes qui leur ont succédé. Ces « anciens", sédentaires, ont pesé davantage sur la vie de leur société, car ils étaient ses éléments stables. Ces compagnons ont nécessairement découvert un jour ou l'autre l'existence des francsmaçons, dont les rituels imprimés, revues, estampes, décors, objets, estampes, diplômes, etc. s'étaient répandus depuis les années 1730 . Nombre de compagnons, dans leurs Mémoires ou au détour d'un poème, renvoient aux classiques de la littérature maçonnique du XIXe siècle (Bazot, Delaulnaye, Clavel, Marconis de Nègre, entre autres $\left.{ }^{16}\right)$.

D'autre part, les obédiences maçonniques se sont ouvertes aux classes populaires, aux ouvriers, aux petits patrons. Dans une même ville, il y eut des loges de bourgeois et d'aristocrates et des loges d'ouvriers et d'artisans, des obédiences qui leur étaient plus fermées et d'autres plus ouvertes. Au sein de ces dernières ont été affiliés des compagnons sédentaires. Ils y ont été attirés par la poursuite d'une fraternité vécue seulement durant le tour, pour s'y instruire, s'ouvrir à d'autres catégories sociales, et accéder à un univers philosophique et spirituel qui leur faisait défaut dans le Compagnonnage.

Ce sont ces lectures, ces découvertes d'objets, ces affiliations à des loges, ces cérémonies dans des temples, ces contacts individuels entre compagnons et francsmaçons, qui ont conduit des compagnons écoutés, respectés et influents à introduire de nouveaux éléments, empruntés à la Franc-maçonnerie, dans leurs Devoirs.

\subsection{Des images de « travailleurs »?}

Il y a un paradoxe à souligner. Ces images émanent et sont destinées à des hommes de métiers mais la représentation des métiers et du travail y est des plus réduites. Une trentaine de métiers sont pourtant présents au sein du Compagnonnage au XIXe siècle. Le secteur du bâtiment groupe les compagnonnages de charpentiers, tailleurs de pierre, menuisiers, serruriers, couvreurs, plâtriers, vitriers... Celui du cuir, les tanneurs, mégissiers, cordonniers, bourreliers, selliers. Le textile a engendré les sociétés de tondeurs de drap, tisserands, teinturiers, tisseurs, cordiers, chapeliers. La métallurgie a donné naissance aux forgerons, cloutiers, maréchaux, poêliers, ferblantiers, fondeurs. Les doleurs (tonneliers), les boulangers, les charrons, les sabotiers et les vanniers ont également leur propre compagnonnage, chacun, comme les précédents, avec son identité rituelle et symbolique, son vocabulaire, ses us et coutumes.

41 Le métier est donc indissociable du compagnon. Il est impossible d'être admis au sein de ces groupements sans être un homme de métier et sans prouver ses capacités, soit en faisant ses preuves à l'atelier ou sur le chantier, soit en confectionnant un « chefd'œuvre » jugé par les autres compagnons.

Et pourtant... Passage obligé pour accéder aux bienfaits du Compagnonnage, le métier n'est pas une fin en soi, un sacerdoce, une obligation à vie. Dès le XIXe siècle (probablement plus tôt encore) on constate que de nombreux compagnons abandonnent leur métier au cours de leur existence, pour devenir épiciers, cafetiersrestaurateurs, hôteliers, fleuristes, représentants de commerce, vignerons ou ostréiculteurs, tandis que d'autres s'engagent dans l'armée à la fin de leur tour de France et y restent jusqu'à leur retraite. Mais ils n'en demeurent pas moins des compagnons jusqu'à la fin de leurs jours. Ils continuent à participer aux assemblées, 
cotisent et votent comme les autres tant qu'ils n'ont pas « remercié » leur société dans les formes prévues. À leur décès, les honneurs leurs sont rendus. L'état civil les enregistre comme sous-officiers, négociants, professeurs, tandis que les compagnons les reconnaissent toujours pour charpentiers, boulangers ou tisseurs. Le lien fraternel issu de l'initiation persiste au-delà du changement d'état.

Ces hommes, impliqués dans leur métier, jusqu'à produire des chefs-d'œuvre inouïs de complexité, peuvent aussi s'en extraire. Car le but du Compagnonnage n'est pas d'enfermer l'artisan dans des gestes mille fois répété, mais, autant que possible, de lui permettre une progression tant professionnelle que sociale et morale. L'élévation emprunte des chemins qui peuvent alors nous surprendre mais qui sont pourtant admises, voire encouragés, par les règles du Compagnonnage.

Le compagnon n'est pas qu'un « travailleur ». Le travail n'est pas une valeur en soi mais un moyen de progresser dans sa vie personnelle et sociale ${ }^{17}$. On conçoit dès lors la réticence que les compagnons ont éprouvée à se fondre au sein des mouvements ouvriers au XIXe siècle. Précurseurs du syndicalisme et de la mutualité, ils s'en sont pourtant tenus collectivement à l'écart. Sélectifs par essence, les compagnonnages ne sont pas des mouvements de masse au sein desquels le dénominateur commun serait l'exercice d'un métier, d'un travail, qu'il soit bien ou mal accompli, et où l'objectif serait prioritairement salarial ${ }^{18}$.

Dès lors, tout comme dans leurs innombrables chansons, la part accordée au métier et au travail est restreintes dans leurs images. Qu'y voit-on? Sur les dessins aquarellés des charpentiers, le métier n'est discrètement figuré que par les outils appendus ici et là : outils emblématiques et non symboliques. Ailleurs, c'est une devise : « Gloire au travail, mépris à la paresse / Le travail et l'honneur, voilà notre richesse. » Sur d'autres, une petite scène nous montre saint Joseph à l'œuvre devant son établi, mais le saint patron est davantage mis en avant que le métier de charpentier. D'autres images aquarellées le confirment : un règlement illustré de tonneliers nous montre, en bas, une toute petite scène de travail; un "souvenir" de couvreur intègre un détail où l'on voit le compagnon grimpant le long d'une corde sur un clocher; sur une image de tanneur, des peaux sont étalées sur une table entourée des outils du métier... On pourrait multiplier les exemples. 
Illustration 5. Maître Jacques, Fondateur des Compagnons du Devoir (Ami de Soubise)

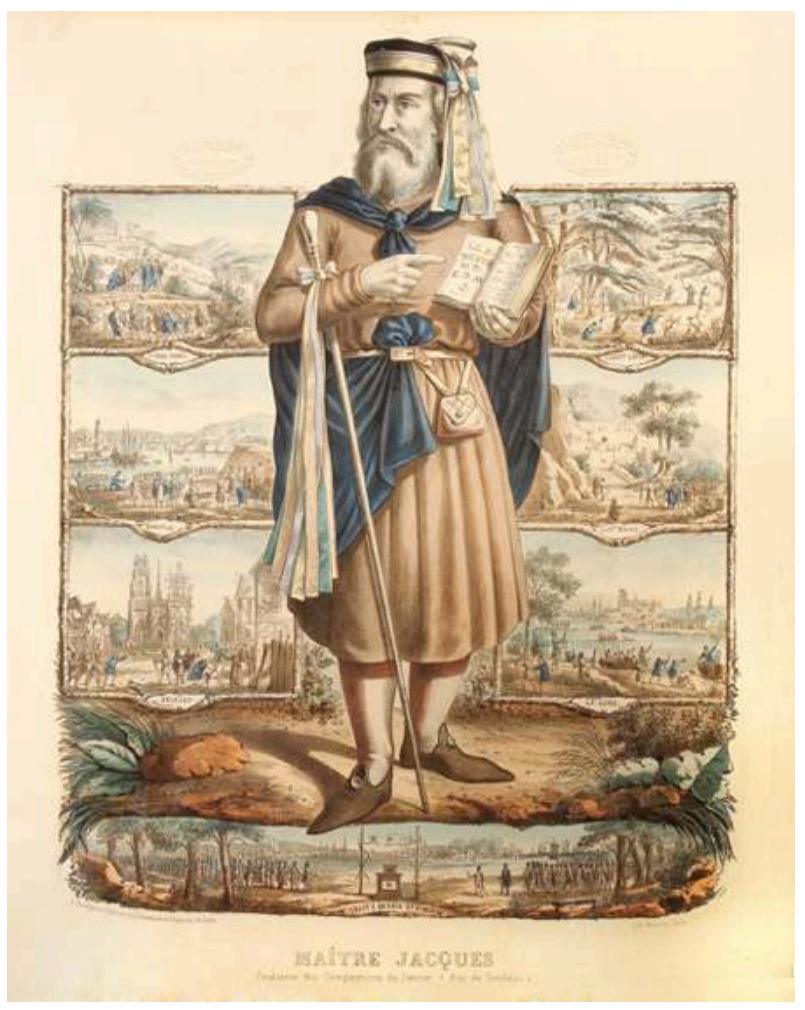

Lithographie, 50 x $40 \mathrm{~cm}$, Agricol PERDIGUIER dit Avignonnais la Vertu, compagnon menuisier du Devoir de Liberté, et MONROCQ, imprimeur-lithographe, Paris, 1863. 


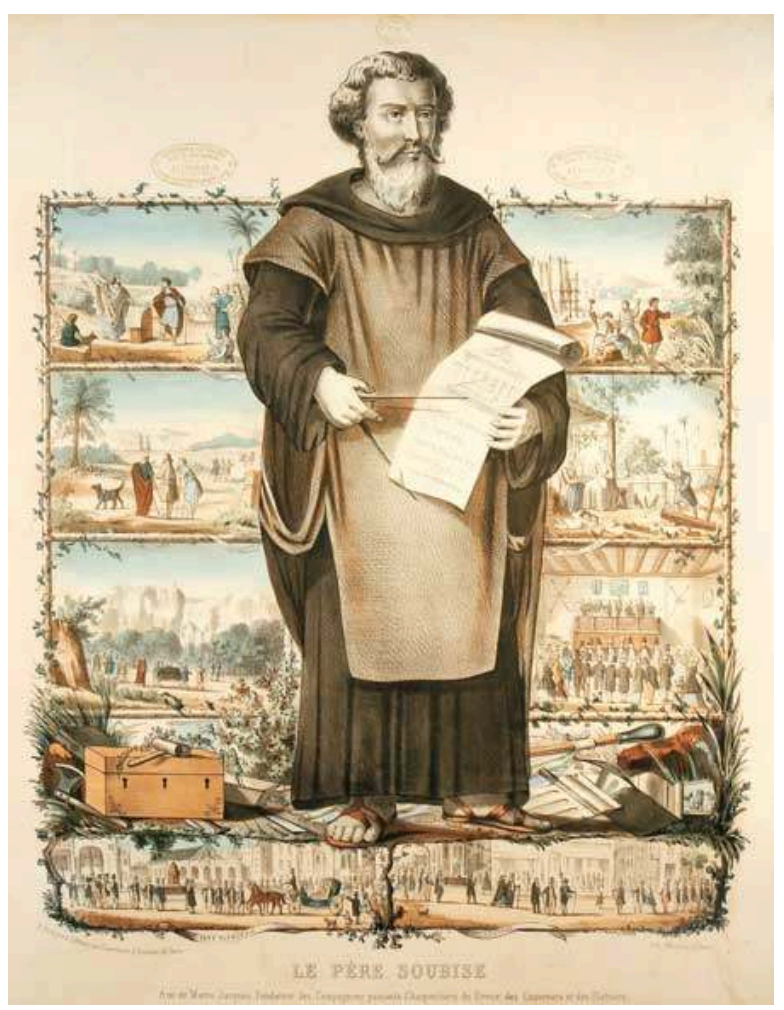

Lithographie, 51 x 41 cm, Agricol PERDIGUIER dit Avignonnais la Vertu, compagnon menuisier du Devoir de Liberté, et MONROCQ, imprimeur-lithographe, Paris, 1865.

En revanche, ces images privilégient les valeurs du Compagnonnage, sous forme d'allégories antiques: Minerve (la sagesse), Thémis (la justice), Hercule (la force). Les mythes fondateurs des Devoirs, les légendes et leur enseignement moral, sont traités au premier plan : le temple de Salomon, le pèlerinage à la Sainte-Baume, Maître Jacques et le Père Soubise, tout un ensemble de figures symboliques et mystérieuses sont présentes sur les lithographies dès les années 1840 .

47 C'est la transcendance du métier par la légende, le rite et le symbole, qui est mise en avant, pas le travail, «valeur » commune aux bons comme aux mauvais ouvriers, dont le compagnon cherche toujours à se distinguer, y compris par sa tenue, son langage, ses mœurs. En témoignent les beaux dessins-souvenirs exécutés sous la Restauration par Leclair, à Bordeaux, où les compagnons qui les achetaient avant de rentrer dans leurs foyers, sont toujours représentés fièrement campés, canne en main, en redingote et haut-de-forme, leurs « couleurs » flottant à la boutonnière ou au chapeau.

\section{3. Évolution}

Les compagnonnages ont été confrontés tout au long du XIXe siècle à l'évolution de leur environnement social, politique, religieux et technique, alors même qu'il perpétue auprès de ses membres, par ses chansons notamment, l'idée d'un Devoir éternel. Les images reflètent les tensions et les adaptations des compagnons à leur contexte. 


\subsection{Une « tradition » perméable} plus anciens et ne s'y substituèrent pas en totalité. Ou bien encore, si les mots, les rites et les images étaient maçonniques, leur signification en fut repensée, déformée et adaptée dans un sens " compagnonnique ». Ces transferts de formes et de mots firent naître des compagnonnages maçonnisés, mais ceux-ci n'en demeurèrent pas moins des compagnonnages de métiers et non des franc-maçonneries ouvrières.

\subsection{Les mécanismes de l'évolution iconographique}

54 Il convient de préciser quelques points encore sur l'évolution des images. Sous la Restauration, les premières étaient empreintes de simplicité car elles ne comportaient qu'un seul thème et quelques éléments emblématiques, qui évoquent le métier, l'honneur, la fierté, la fraternité, qui sont inspirés et protégés par Dieu et le saint patron du métier. 

moins de place dans l'image et deviennent secondaires. Les outils emblématiques du métier sont recomposés en des « blasons » renforçant l'identité de chaque société. Dans le même temps, ils passent du rang d'emblèmes à celui de symboles, car certains métiers qui ne les emploient pas les intègrent cependant avec ceux de leur art (le compas et l'équerre, par exemple, sont associés au rouable et à la pelle des boulangers). Ou bien, mêlés à ceux d'autres métiers, ils sont déversés par des cornes d'abondance. Ils n'expriment plus alors le métier d'un corps mais celui de tous les compagnons, dans l'optique d'un Compagnonnage réunifié, selon les désirs pacifiques de Perdiguier.

Illustration 7. L'union des corps d'États

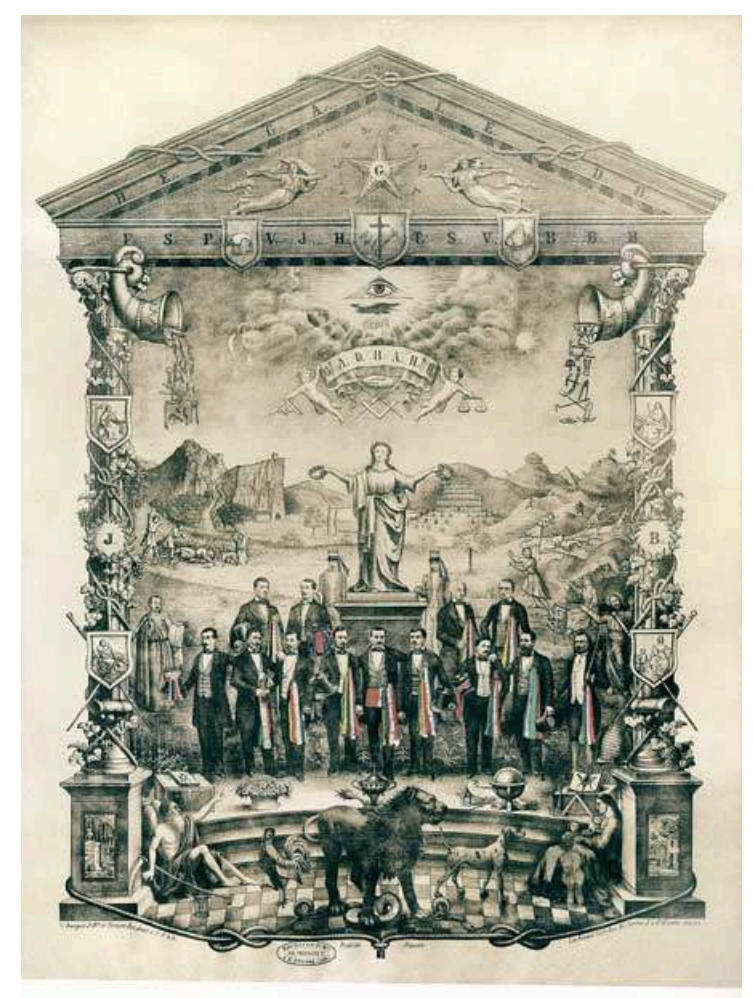

Lithographie, 80 × 56 cm, Jean-Baptiste BOURGUET dit Forézien Bon Désir, compagnon tisseurferrandinier du Devoir, et PINSARD, imprimeur-lithographe, Saint-Etienne, entre 1870 et 1880.

Aux figures des angelots et du saint patron évoquant le sacré, s'ajoutent ou se substituent d'autres représentations de même portée, mais plus tout à fait de même sens. Ainsi apparaissent des dédicaces telles que «A la gloire du Grand Architecte de l'Univers ", isolées ou conjointes avec «Gloire à Dieu », dont la première est issue de la Franc-maçonnerie. Le sens, le ressenti surtout, de chacune d'elles est similaire mais pas identique. Se répandent aussi des motifs tels que le triangle en gloire, avec ou sans œil au centre.

Glissement de sens également à propos du portique de temple abritant le compagnon en pied des premières images. Il n'est alors qu'un élément majestueux de décor, théâtralisant la position du fier compagnon avec sa canne et ses couleurs et évoquant les arts auxquels il contribue par son métier. Peu à peu, certains corps éditent des lithographies où le portique subsiste mais avec un sens différent, puisque ces deux colonnes sont désormais parées des lettres $\mathrm{J}$ et $\mathrm{B}$, qu'une étoile à cinq branches brille au 
fronton et qu'un dallage de carreaux noirs et blancs se déploie entre les piédestaux. Il s'agit désormais du temple de Salomon, de ses colonnes Jakin et Boaz et du "pavé mosaïque " (damier) empruntés à la symbolique maçonnique, comme on le constate avec la lithographie de Bourguet sur L'Union des corps d'états. Le décor évolue encore avec Le Génie du Compagnonnage, où les colonnes deviennent des cèdres du Liban, renvoyant, eux aussi, à la construction du temple de Salomon.

\subsection{L’apport des images extérieures au Compagnonnage}

L'évolution de ces images provient aussi de ce que les compagnons y intègrent des représentations issues d'estampes non compagnonniques. Ainsi, lorsque Perdiguier édite son Père Soubise (1865), il copie manifestement l'illustration d'un ouvrage maçonnique où figure la scène de Salomon invitant son architecte à édifier un temple à la gloire du Très-Haut. De même, la vue de la Sainte-Baume figurant sur la lithographie de Maître Jacques est issue d'une image populaire de son temps. Bourguet fera de même lorsqu'il composera son Voyage de la Sainte-Baume (c. 1880). Quant au sabotier Guépin Coeur d'Amour, il insère en haut de son estampe dédiée aux Enfants du Devoir des représentations de Moïse et de David issues d'une bible du XVIIe siècle dont la page de titre a été gravée par Pierre Firens. Bourguet encore, dans L'Union des corps d'états, reproduit presque à l'identique un détail d'une estampe maçonnique et s'inspire du fronton du Panthéon pour représentant la Gloire distribuant ses couronnes aux valeureux compagnons. Quant au cordonnier Charue, il «compagnonnise » pour Le Génie du Compagnonnage une image républicaine de 1848 qui devient le char sur lequel les trois fondateurs font le tour du globe ${ }^{22}$.

Illustration 8. Dédié aux enfants du devoir

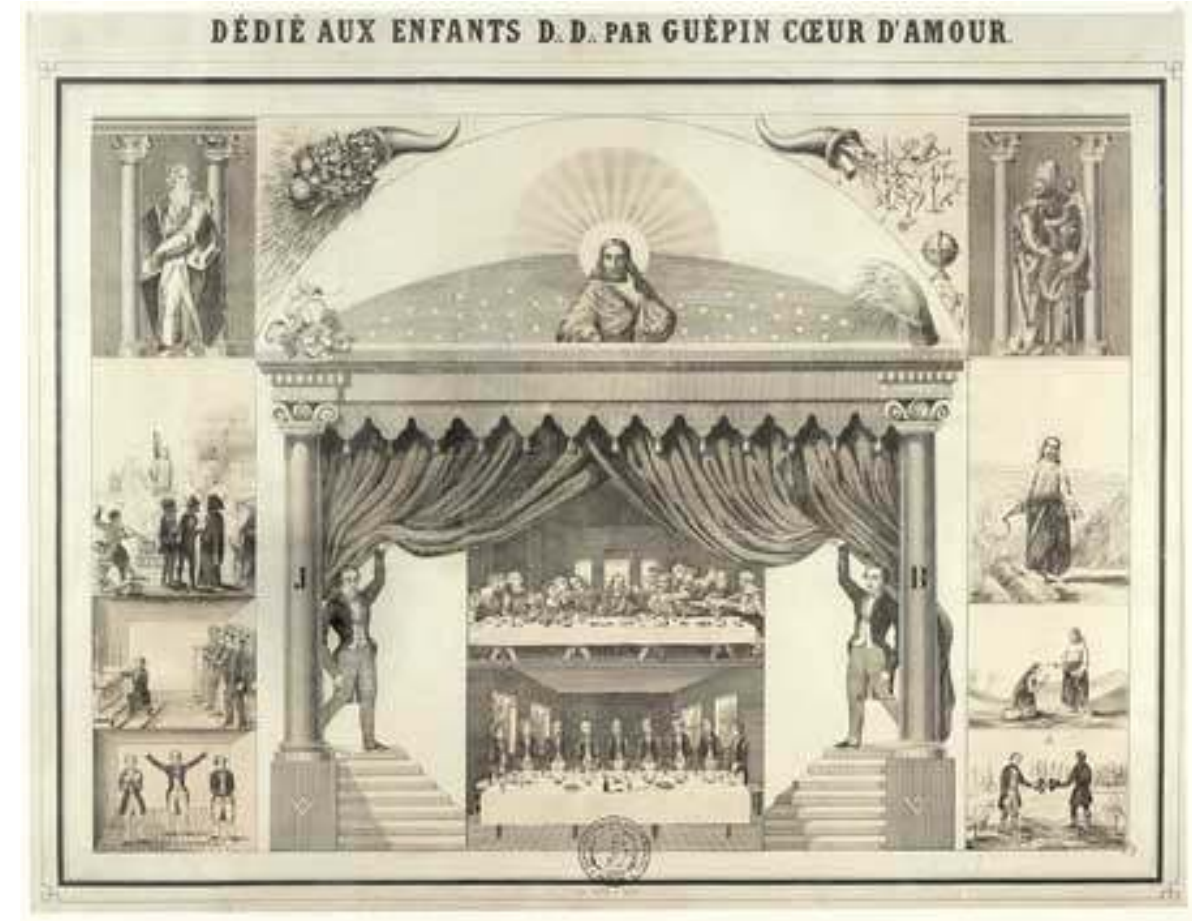

Lithographie ; 50 × 63,5 cm ; auteur : Auguste AMAND dit Guépin Cœur d'Amour, compagnon sabotier du Devoir, et JACOB, imprimeur-lithographe ; Orléans ; c. 1850-1860. 


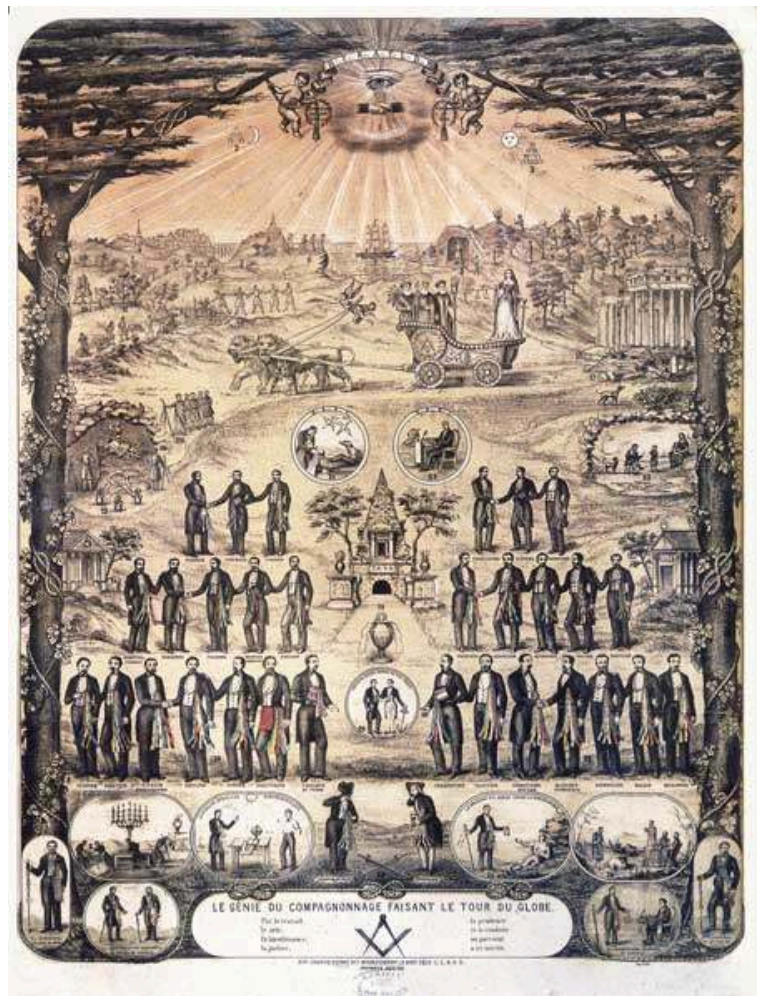

Lithographie, 71 × $51 \mathrm{~cm}$, Pierre CHARUE dit Bourguignon le Bien Zélé, compagnon cordonnier-bottier du Devoir, et FRICK frères, imprimeurs lithographes, Paris, 1882, 2e éd. 1890.

Ces compagnons ont non seulement emprunté à des images antérieures, religieuses, maçonniques ou profanes, mais se sont copiés les uns les autres. On sait que la représentation des trois fondateurs Salomon, Jacques et Soubise, par Perdiguier, est à l'origine de modèles auxquels les autres compagnons se réfèrent aujourd'hui encore. Pour sa part, Charue s'est inspiré, pour composer Le Génie, de L'Union des corps de Bourguet. Et Contios, un charpentier d'Agen, éditera en 1880 une estampe qui reprendra sans vergogne les vignettes du tableau du Père Soubise (1865) de Perdiguier.

Ce processus d'imitation et d'ajouts progressifs est à l'image de l'évolution rituelle et symbolique du Compagnonnage, qui, par petites touches, a transformé sur un siècle un Devoir qu'ils ont affirmé vouloir transmettre dans toute sa " pureté originelle ».

Un point qu'il serait intéressant d'approfondir (mais le pourra-t-on faute de sources ?) est celui de la part des apports due aux dessinateurs non-compagnons. Les typographes, ouvriers qualifiés et instruits, étaient assez nombreux dans les loges maçonniques au XIXe siècle et ils ont pu introduire, de leur propre chef, tel ou tel motif symbolique ou des détails d'estampes qu'ils connaissaient bien pour les avoir dessinées pour leurs frères maçons ou vues dans leur loge. Leurs liens avec les compagnons étaient par ailleurs assez étroits puisque Perdiguier, dans le Livre du compagnonnage, écrit qu' « une partie des typographes tend à se constituer en compagnonnage $»^{23}$.

Compte tenu de tous ces apports extérieurs et des innovations propres à chaque compagnon éditeur, le contenu des lithographies de la seconde moitié du XIXe siècle sera fort éloigné du seul Devoir de chaque corps. Les acheteurs de ces images ne pouvaient plus les comprendre en se référant aux informations communiquées lors de 
leur réception. C'est pourquoi les éditeurs en vinrent à imprimer des notices explicatives fournies en même temps que leurs estampes: Bourguet, Charue, Fradin, entre autres. Ils avaient constitué leur propre langage symbolique et des cryptographies dont eux seuls avaient la clef. La seule confrontation avec le Devoir de telle ou telle corporation ne suffit plus, aujourd'hui, faute d'avoir retrouvé ces notices, pour décrypter les multiples initiales triponctuées ou les symboles semés à profusion sur certaines estampes.

63 L'évolution du contenu des estampes est aussi celle du passage d'un symbolisme collectif à un symbolisme destiné à "une élite dans l'élite ». Les compagnons, qui s'estimaient déjà des initiés détenteurs de mystères au sein des autres ouvriers, instituèrent eux-mêmes des grades supérieurs au sein de leurs propres sociétés. Le cas des compagnons toiliers du Devoir, diffusant leur lithographie à une partie seulement d'entre eux et leur fournissant des notices plus ou moins détaillées selon leur degré d'initiation, illustre la tendance du Compagnonnage du XIXe siècle à élaborer des hiérarchies secrètes en son sein, à l'instar de la multiplication des « hauts grades » au sein des obédiences maçonniques.

\subsection{De l'estampe à la photographie}

L'ultime évolution des images a été celle que provoqua la photographie. Inventée au temps des dessins aquarellés, elle ne prend son essor que sous le Second Empire. Elle aussi permet la création d'images qui coexistent durant près d'un demi-siècle avec la lithographie. A priori, les photographies n'ont pas la même fonction que les estampes lithographiées. Ces dernières sont décoratives et elles sont le support d'idées, voire des outils de réflexion et d'instruction pour qui les contemplent. Ce sont aussi des souvenirs du tour de France. Et pourtant, les photographies vont peu à peu les concurrencer. 


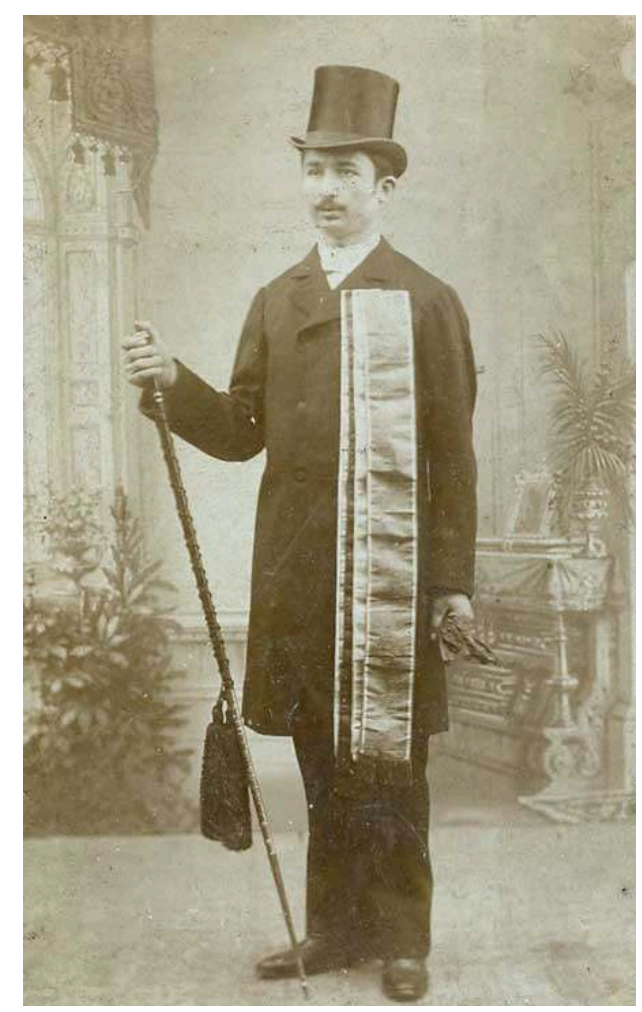

Photographie-portrait, Tours, c. 1900.

D'une part, elles vont remplir la fonction originelle des portraits en pied que les compagnons faisaient peindre par Leclair ou Lemoine en passant à Bordeaux ou Angers. Désormais, après leur réception, ils seront très nombreux à se faire photographier et à disposer de leur portrait en redingote, gibus, canne et couleurs, le plus souvent en petit format carte de visite. Les délais sont courts, le prix modique et le compagnon peut obtenir son «souvenir du tour de France » auprès de plusieurs photographes dans une même ville.

D'autre part, la photographie permet de conserver le souvenir de manifestations collectives : assemblées, excursions, chantiers mémorables, grands chefs-d'œuvre mais surtout groupes de compagnons posant autour de leur Mère lors des fêtes patronales. Les portraits et les photos de groupes seront innombrables dès les années 1860 et sont toujours exécutés sans discontinuité, de fête en fête, partout en France.

La photographie introduit un retour au réalisme qui avait quelque peu disparu lors du passage du dessin à la lithographie. Avec elle, plus de symboles ni d'épisodes légendaires. Ces derniers ne disparaîtront jamais totalement au sein des compagnonnages mais dès les premières années du XXe siècle, quelques-unes seulement des dizaines d'estampes du demi-siècle écoulé continueront à être imprimées, soit avec les mêmes pierres, soit par d'autres procédés de reproduction. Jusque dans l'entre-deux-guerres, chez Audebaud à Saint-Maximin on trouvait encore Jacques et Soubise, les Mères de quelques corps disposaient encore de quelques exemplaires d'images diverses, les boulangers du Devoir ainsi que les charpentiers du Devoir de Liberté continuaient à délivrer des diplômes imprimés quarante ans plus tôt. Ces images-là perpétuaient les mythes fondateurs. Mais il n'y eut plus, ou très peu, de compagnons qui éprouvèrent le besoin d'imaginer de nouvelles 
compositions et de fixer par l'image leurs rites, symboles et mythes associés à leurs contemporains.

\subsection{Des représentations figées à la fin du XIXe siècle}

En effet, ces compagnons en redingote et haut de forme paraissent aujourd'hui appartenir au passé alors qu'ils représentent ceux qui ont commandé leur portrait ou composé l'estampe. Leur tenue est contemporaine de l'image. Mais ces compagnons du XIXe siècle sont pourtant figurés sans rien qui les sépare des scènes de la construction du temple de Salomon ou de l'assassinat de Maître Jacques. Le présent et le passé mythique coexistent, l'un prolongeant l'autre.

Or, sur les rares images du XXe siècle, non seulement la dimension symbolique et légendaire est discrète, mais surtout, elle n'est plus mise en relation avec le Compagnonnage contemporain. Et lorsqu'il s'agit d'évoquer le passé des Devoirs, les dessinateurs ont systématiquement recours au compagnon en redingote et gibus, canne en main, couleurs au vent, façon Ardéchois Cour Fidèle. Ou bien, franchissant un demimillénaire, les illustrateurs représentent l'ancien compagnon sous les traits du bâtisseur de cathédrales, comme si celui de l'Ancien Régime n'avait jamais existé (il n'a d'ailleurs laissé aucune trace dans leur imaginaire).

Illustration 11. Groupe de compagnons charpentiers du Devoir réunis pour la Saint-Joseph 1926 à Tours, cour de l'hôtel de la Croix-Blanche.

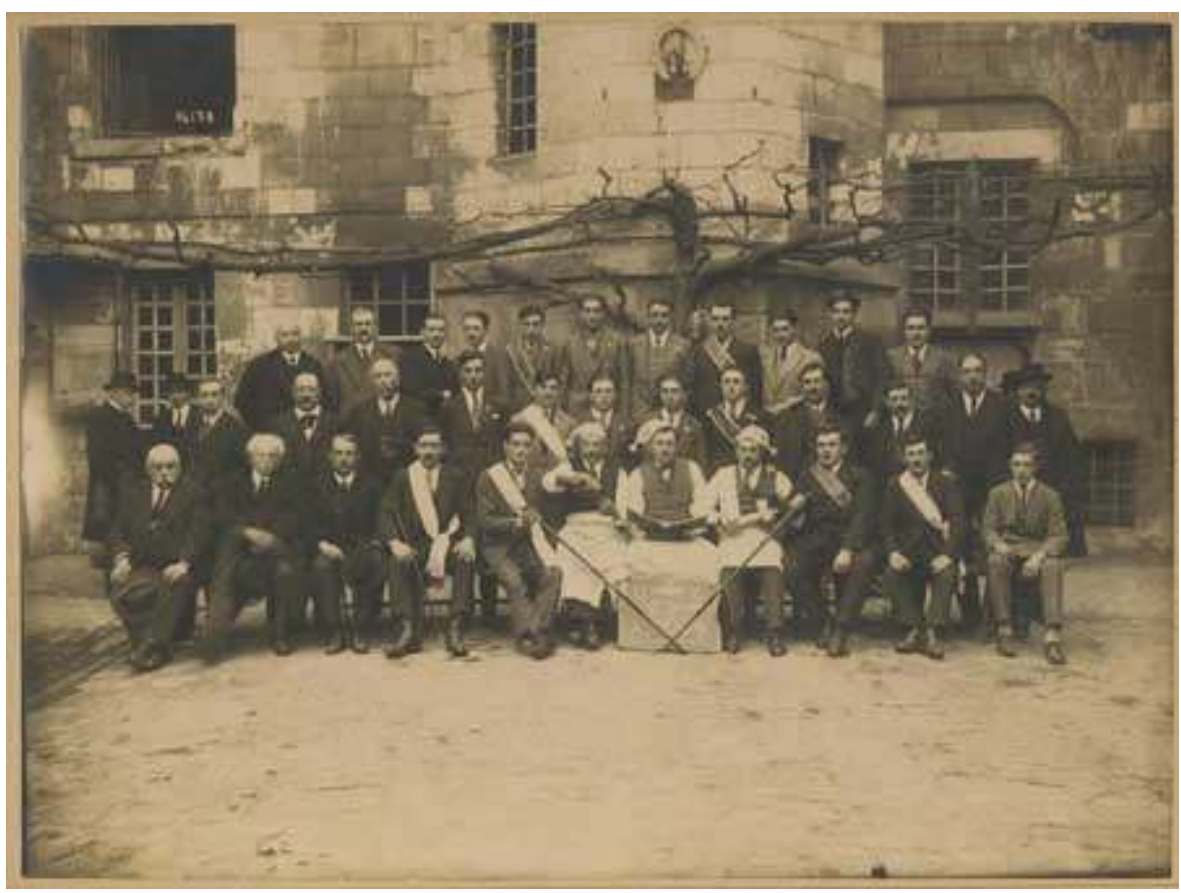

Photographie, Tours, 1926

Aucune image ne représente un compagnon mécanicien en bleu de travail, la casquette sur la tête, en veste et cravate, ou même en jean et baskets, devant les pyramides d'Égypte, ou posant sur fond de meurtre d'Hiram. Et pourtant, il s'agirait de l'exacte réplique, adaptée à notre époque, des représentations du XIXe siècle. Une telle association semblerait grotesquement anachronique et ridicule, parce que les compagnons d'aujourd'hui ne croient plus à la réalité des trois fondateurs, ils ne se 
sentent plus leurs héritiers directs et souvent même n'ont qu'une connaissance superficielle des légendes qui y sont attachées. La transmission de cette étroite relation entre le passé mythique et la vie du compagnon s'est en effet mal opérée après 1950. Dès lors, cette rupture ne permet plus guère aux jeunes compagnons de comprendre les estampes du XIXe qui ornent leurs sièges.

\section{Conclusion}

71 Le déclin des estampes dans le monde compagnonnique correspond aussi au déclin d'une technique. La lithographie n'est plus guère utilisée par les imprimeurs dès avant 1914. Il n'y a pas eu non plus de report du contenu des images compagnonniques sur d'autres supports, via une technique d'impression différente. On peut donc se demander si cette floraison d'images si particulières ne correspondrait pas à une étape transitoire entre un compagnonnage qui fonde sa transmission sur l'oralité et un autre sur l'écrit. En effet, on ne connaît pas l'équivalent des dessins aquarellés de Leclair et Lemoine avant la Révolution. Sans préjuger de découvertes à venir, à ce jour les portraits en pied, les souvenirs du tour et les conduites sont inexistants sous l'Ancien Régime. Pourtant, le dessin aquarellé existait bien chez les compagnons, et de longue date : on en connait de très beaux exemplaires chez les compagnons passants tailleurs de pierre, dès les années 1720 et tout au long du XVIIIe siècle, à Paris, Chalon-surSaône, Avignon, Marseille et Bordeaux. Mais il s'agit là d'en-têtes de rôles et non de souvenirs individuels. L'usage de ces images est collectif et elles sont commandées par une société à l'un des siens habile à dessiner et à peindre.

L'ancien Compagnonnage est caractérisé par la pratique intense de la mémoire des rites et la transmission orale, et quand il y a écrit et image, ils sont destinés au fonctionnement de la société compagnonnique, pas à l'usage individuel. Après la Révolution, l'image vient combler le besoin de souvenirs et d'identité, car les compagnons deviennent des marginaux au sein du monde ouvrier. Les "travailleurs " du XIXe siècle les considèreront d'ailleurs, de plus en plus, comme des vestiges attardés de l'Ancien Régime. L'image leur convient car elle est la projection de leurs idées et de leur originalité. De plus, elle s'adresse à une population qui maîtrise encore mal l'écriture.

Mais l'évolution des compagnons se poursuit par leur alphabétisation croissante. Ce ne sera désormais plus par l'image mais par l'écrit que les compagnons affirmeront leur originalité. Dès 1839, Perdiguier publie son Livre du Compagnonnage et d'autres le suivront, par des ouvrages réformateurs, des recueils de poésies et des Mémoires (le peintre Deruineau en 1850, le boulanger Arnaud en 1859, le cordonnier Guillaumou en 1864). À la fin du siècle, la diffusion de journaux spécifiques aux compagnons atteste que tous ou presque savaient lire. C'est désormais avec ce support que s'opérera la diffusion de leurs idées et son irruption coïncide avec le déclin des estampes. Après 1880 , l'écrit se substitue de plus en plus à l'image et va la supplanter à l'aube de la Grande Guerre. 


\section{BIBLIOGRAPHIE}

Adell-Gombert (2008) Des hommes de Devoir, les compagnons du Tour de France (XVIIIe-XXe siècle), Paris, Éd. de la Maison des sciences de l'homme, 2008.

Bastard L. (2006), « Les sources méconnues du Compagnonnage français au XIXe siècle, I : Des Compagnons du Silence aux Compagnons tanneurs-corroyeurs du Devoir », in Renaissance traditionnelle, $\mathrm{n}^{\circ} 145$, p. $73-87$.

Bastard L. (2010), Images des Compagnons du Tour de France, Paris, J.-C. Godefroy.

Bastard L. (2012), « Rites et symboles des compagnons : une construction permanente », in Fragments d'histoire du Compagnonnage, volume 14, Tours, musée du Compagnonnage.

Coornaert E. (1966), Les Compagnonnages en France du Moyen Âge à nos jours, Paris, Les Éditions ouvrières.

Lecotté R. (1948), « Essai pour une iconographie compagnonnique : « champs de conduite » et « souvenirs » du Tour de France », in Artisans et paysans de France, Strasbourg-Paris, F.-X. Le Roux $\&$ Cie.

Martin Saint-Léon E. (1977) (rééd. 1901), Le Compagnonnage, son histoire, ses coutumes, ses règlements et ses rites, Paris, A. Colin, Librairie du Compagnonnage.

Philippon J. (2013), « Les rites et les Devoirs », in Fragments d'histoire du Compagnonnage, volume 15, Tours, musée du Compagnonnage.

Philipppon J. (2014), « Les savoirs antiques dans le Compagnonnage », in Fragments d'histoire du Compagnonnage, volume 16, Tours, musée du Compagnonnage.

\section{NOTES}

1. MARTIN SAINT-LÉON Étienne : Le Compagnonnage, son histoire, ses coutumes, ses règlements et ses rites; Paris, A. Colin, 1901, rééd. Librairie du Compagnonnage, 1977, p. 270.

2. COORNAERT Émile: Les Compagnonnages en France du Moyen Âge à nos jours; Paris, Les Éditions ouvrières, 1966, p. 245.

3. LECOTTÉ Roger : «Essai pour une iconographie compagnonnique : « champs de conduite » et « souvenirs » du Tour de France », in : Artisans et paysans de France, Strasbourg-Paris, F.-X. Le Roux \& Cie, 1948 (tiré à part).

4. BASTARD Laurent: Images des Compagnons du Tour de France; Paris, J.-C. Godefroy, 2010.

5. Après les bouleversements de la Révolution (abolition des communautés de métiers par la loi d'Allarde et interdiction des associations d'ouvriers par la loi Le chapelier, en 1791, mais aussi fin de la monarchie, atteintes à l'Eglise, conscription), les compagnonnages s'efforcent dès les années 1800 de rénover leurs rites et mettent en place, lors d'assemblées générales de tous corps, et non sans mal, des tableaux généalogiques définissant leurs droits de préséance respectifs. Ces généalogies, mi-historiques, mi-légendaires, ne concernent que les corps de métiers antérieurs à la Révolution et sont fréquemment contestées jusque dans les années 1880 . Or, dès 1808 , les cordonniers se constituent en compagnonnages, suivis des sabotiers (1809), des boulangers (1810), des tisseurs-ferrandiniers (1831), d'où émanent à leur tour des associations dissidentes. Ces sociétés ne sont pas reconnues comme légitimes par leurs aînées, qui leur dénient le titre de compagnon, les chassent des villes où elles s'établissent, les maltraitent. En réaction, ces 
compagnonnages tard venus n'ont de cesse de s'affirmer comme de véritables Devoirs en cherchant à se faire " reconnaître " par au moins un corps qui convaincra les autres de faire de même. Tous les moyens sont mis en branle pour convaincre les sociétés anciennes de leur authenticité compagnonnique : démonstrations publiques (cortèges), convocations d'assemblées, invention de filiations remontant jusqu'à la fondation du temple de Salomon ou à l'ancienne Egypte, fabrication d'actes de fondation sur parchemin et en écriture gothique...

6. Les compagnonnages sont des sociétés de type initiatique, fondées sur la transmission de valeurs exprimées par des rites et des symboles. Tout compagnon nouvellement reçu se ressent comme dépositaire d'un patrimoine immuable et pense que ses prédécesseurs ont tous été reçus selon le même rituel que lui. La cérémonie de réception est censée se répéter invariablement comme dans un éternel présent. Or, l'étude des rites et des symboles compagnonniques montre qu'ils ont constamment évolué au fil des décennies, surtout à partir du XIXe siècle et continuent à se transformer de nos jours. Cela, un compagnon qui vient d'être reçu ne le sait pas et il sera nécessairement enclin, par fidélité à son Devoir, à respecter ce qui lui a été transmis. Il existe chez les jeunes reçus un profond respect des «anciens " et des "antiquités » (le patrimoine compagnonnique fait de légendes, rites et symboles), qui conduit à adopter avec obéissance les innovations qui pourtant été régulièrement introduites avant eux par les "anciens". Le phénomène a pu se produire aussi en ce qui concerne l'achat de dessins aquarellés puis de lithographies, devenu "de tradition » au passage de certaines villes (dont Bordeaux et Lyon), simplement parce que les prédécesseurs des jeunes gens qui passaient dans ces villes avaient ainsi fait. Sur ce qu'Émile COORNAERT appelle "l'influence croissante des anciens ", cf. Les Compagnonnages, p. 102-104. Sur l'évolution des rites, cf. L. BASTARD : « Rites et symboles des compagnons : une construction permanente ", in : Fragments d'histoire du Compagnonnage, volume 14 ; Tours, musée du Compagnonnage, 2012 et J. PHILIPPON : «Les rites et les Devoirs ", in : Fragments d'histoire du Compagnonnage, volume 15 ; Tours, musée du Compagnonnage, 2013.

7. Les portraits-souvenirs représentent le compagnon en pied, au centre du dessin, sous un portique de temple, paré de ses «couleurs" (rubans, attributs spécifiques de chaque société compagnonnique) et tenant sa canne ; il est accompagné d'un décor de fleurs, d'outils et de son saint patron. Les conduites le montrent suivi d'un cortège de compagnons de son métier, qui le suivent jusqu'aux portes de la ville qu'il quitte pour une autre et parfois à la fin de son tour de France. Il s'agit d'un rite caractéristique des compagnonnages. En arrière-plan sont toujours représentés les monuments de la ville, un pont, un fleuve, des navires et de petites scènes de la vie quotidienne. Ces deux types de dessins aquarellés sont légendés par Leclair et Lemoine "souvenirs" et "champs de conduite», suivis du surnom du compagnon, de la date de sa réception, de celle de son départ et de la ville où il se rend.

8. Le doleur était l'ouvrier qui confectionnait les douelles (ou douves) en refendant avec une hache appelée doloire les planches (le merrain) fournies par les bûcherons. Ces planches, finies à la plane (couteau à deux manches) puis biseautées, étaient assemblées pour former un fût par le tonnelier. Ces opérations sont largement mécanisées depuis le milieu du XXe siècle.

9. Catégorie qui n'est composée presque exclusivement que de lithographies. Les gravures sur cuivre ou acier, au burin ou à l'eau forte, sont très rares et concernent seulement, à notre connaissance, les "affaires" compagnonniques (passeports, marques secrètes, actes de réception, certificats de remerciements et quelques diplômes de fin de tour).

10. Agricol PERDIGUIER, dit Avignonnais la Vertu (1805-1875) se lança dans l'édition de lithographies compagnonniques à son retour en France, après quatre années d'exil forcé en Suisse du fait de ses convictions de député républicain. Il édita les quatre planches du Compagnonnage illustré (1858), La Réconciliation des compagnons (1862), Salomon (1862), Maître Jacques (1863) et Le Père Soubise (1865). Jean-Baptiste BOURGUET, dit Forézien Bon Désir (1827-1900), établi à Saint-Etienne, édita entre 1875 et 1900 les lithographies connues sous le titre de Voyage de la Sainte-Baume, L'Union des corps d'états, Le Devoir en boutique, Maître Jacques, Honneur aux hommes 
d'élite, Le Devoir des compagnons tisseurs-ferrandiniers, La Fidélité, un portrait du compagnon tanneur Pierre Romian et deux autoportraits.

11. Le Ralliement des compagnons du Devoir insère entre 1885 et 1900 les annonces du tisseur J.-B. Bourguet et consacre de petits articles à la réédition de ses estampes. L'Union Compagnonnique, entre 1890 et 1898, publie les annonces des compagnons Fardin et Charue, auteurs du Temple compagnonnique et du Génie du Compagnonnage faisant le tour du Globe.

12. Il s'agit de la grande lithographie $(77 \mathrm{~cm}$ x $63 \mathrm{~cm})$ de Raphaël RIBIÈRE dit La Sincérité de Villeneuve-de-Berg, dessinée par J. BÉROT et imprimée par KAEPPELIN et Cie.

13. Ce poème en dix couplets est daté de «Besançon, 25 février 1888 » et figure dans : Poésies; Feuilles et profils ; Besançon, 1891, p. 157-160.

14. COORNAERT É., op. cit., (sur la réception), p. 154 ; ADELL-GOMBERT : Des hommes de Devoir, les compagnons du Tour de France (XVIIIe-XXe siècle), Paris, Éd. de la Maison des sciences de l'homme, 2008, p. 175 ; BASTARD L. « Rites et Devoirs... », p. 199-215.

15. Amorcé dans les années 1880 par les compagnons du Devoir représentant le courant conservateur du Ralliement, face à la Fédération puis à Union Compagnonnique (1889), qualifiées de "franc-maçonneries ouvrières", l'antimaçonnisme culmine durant l'Occupation sous la plume de Jean BERNARD, le fondateur de l'Association ouvrière des compagnons du Devoir (1941). Ce dernier publia un chapitre particulièrement révélateur de son hostilité envers les institutions maçonniques dans Le Compagnonnage, rencontre de la jeunesse et de la tradition; Paris, P.U.F., 1972, p. 84-88: "De l'indépendance du Compagnonnage par rapport aux associations maçonniques. Ne pas succomber à la tentation des faux secrets. ». François ICHER a analysé les raisons de ces positions et les nuances qu'elles ont connues au fil des ans et selon les mouvements compagnonniques dans Les Compagnonnages en France au XXe siècle; histoire, mémoire, représentations ; Paris, J. Grancher, 1999, p. 253-267 : «Compagnonnage et franc-maçonnerie : la permanence d'une confusion ».

16. BAZOT : Manuel du franc-maçon (1817), DELAULNAYE, Thuileur de l'Ecossisme (1821), CLAVEL : Histoire pittoresque de la Franc-maçonnerie (1843), MARCONIS DE NEGRE : Le Rameau d'or d'Eleusis (1861), sont des auteurs cités par Agricol PERDIGUIER dans Le Livre du Compagnonnage (1841), Pierre CAPUS, compagnon cordonnier, dans la Lyre compagnonnique (1856), Toussaint GUILLAUMOU, autre compagnon cordonnier, dans ses Confessions d'un compagnon (1864). Les compagnons sabotiers du Devoir ont repris mot à mot des passages du Thuileur pour écrire leur Livre de Devoir.

17. Se considérant comme une élite ouvrière par leur qualification professionnelle et pour avoir été élevé au-dessus du «vulgaire » par leur initiation, la majorité des compagnons aspiraient à devenir des chefs d'entreprises, à sortir du rang, à acquérir une respectabilité dans leur ville. On ne compte pas aux XIXe et XXe siècles ne nombre de compagnons qui s'établirent à leur compte, occupèrent des mandats municipaux et départementaux, participèrent à l'activité des organismes professionnels, furent médaillés de la Mutualité, ou encore s'investirent dans les corps de sapeurs-pompiers et des associations locales de toute nature. Dans les années 1990, une enquête conduite par l'Association ouvrière des compagnons du Devoir permettait de constater que près de $50 \%$ de ses membres avaient fondé leur entreprise, les autres occupant des postes de cadres ou d'enseignants.

18. ICHER, op. cit., p. 84-90: Le problème des syndicats; p. 282-300: Compagnonnage et syndicalisme : complexité et relativité d'une opposition.

19. BASTARD L.: «Rites et symboles des compagnons : une construction permanente", in : Fragments d'histoire du Compagnonnage, volume 14 ; Tours, musée du Compagnonnage, 2012.

20. PHILIPPPON J. : "Les savoirs antiques dans le Compagnonnage », in : Fragments d'histoire du Compagnonnage, volume 16 ; Tours, musée du Compagnonnage, 2014. 
21. BASTARD L. : «Les sources méconnues du Compagnonnage français au XIXe siècle, I : Des Compagnons du Silence aux Compagnons tanneurs-corroyeurs du Devoir», in: Renaissance traditionnelle, $\mathrm{n}^{\circ} 145$, janvier 2006, p. 73-87.

22. Sur les sources d'inspiration de toutes les estampes précitées, cf. BASTARD L.: Images des Compagnons du Tour de France.

23. Edition de 1841, p. 96. Les typographes n'intègreront l'Union Compagnonnique qu'en 1894.

\section{RÉSUMÉS}

Les compagnonnages sont des associations fraternelles de type initiatique rassemblant de jeunes ouvriers d'une trentaine de métiers différents. Elles se sont constituées à la fin du Moyen Age dans un triple but d'assistance mutuelle, d'éducation et de perfectionnement professionnel. Elles favorisent le voyage de ville en ville (le tour de France) durant quelques années. Elles ont perduré jusqu'à nos jours mais ont dû s'adapter aux transformations de leur environnement politique, social, religieux et technique. Au cours du XIXe siècle, les compagnonnages ont eu recours aux images (dessins aquarellés et lithographies) pour se représenter. Leurs fonctions ont évolué tout au long de cette époque, passant du simple souvenir du tour de France à un ensemble complexe de symboles et de légendes constituant un enseignement crypté à destination des compagnons. Ces images ne constituent pas des "images du travail" puisque les métiers y sont peu évoqués, mais mettent surtout en avant les fonctions morales et éducatives de l'institution compagnonnique. Ce texte explore la piste d'un idiome figuratif des groupes professionnels, qui serait composé non de façons de parler, de valeurs, d'habitudes et croyances, mais d'images et de codes visuels. D'une certaine façon, tout groupe professionnel se trouve confronté une fois ou l'autre à la nécessité de se doter d'un moyen visuel de symboliser son activité, de manière qui soit à la fois reconnaissable par autrui et acceptable ses membres, voire qui soit susceptible de favoriser l'identification mutuelle et la cohésion du groupe. La solution à ce problème est souvent trouvée dans la représentation des outils du métier (ou de certains attributs de l'activité), qui fonctionnent ainsi de manière métonymique comme représentation du professionnel lui-même, et le cas échéant du groupe dans son ensemble. Ce procédé, qui semble dans une large mesure spécifique aux groupes professionnels, se retrouve dans des communautés très éloignées dans le temps et les cultures, des scribes égyptiens aux artisans du Moyen-Age ou aux professions libérales qui défilent contre la loi Macron. L'article tente de baliser, d'un côté, les fondements structurels de cet idiome (les manières dont un objet ou outil est transformé en emblème ou symbole d'un être collectif), et, d'autre part, ses contenus caractéristiques, qu'il relie à une volonté d'affirmation de soi des groupes, à la quête du salut religieux, ou à une façon plus polémique de s'auto-représenter qui se retrouve dans les surenchères de glorification du métier, ou à travers la mise en scène des outils ou attributs dans le cadre de l'action collective.

This article follows the trail of the figurative dialect of professional groups, made up of pictures and visual codes rather than idioms, values, habits and beliefs. In some ways, any professional group has to develop at some point a visual mean of representing its own activity. This visual symbol must be both recognizable by outsiders and acceptable for its members, and even capable of fostering mutual identification and group cohesiveness. The solution to these prerequisites is often found in the representing of tools of the trade (or other attribute of the activity), which functions metonymically as a representation of the professional itself or the whole profession. 
This process, which seems generally specific to professional groups, can be observed in old communities of various cultures, from Egyptians scribes to Middle-Ages craftsmen or contemporary professions striking against Macron's bill. This article tries to delimit the structural basis of this dialect (the ways in which an object or a tool is made up to be a symbol or emblem of a collective), and its characteristic contents linked to a wish for assertiveness from groups, a pursuit of religious salvation, or a more controversial to represent itself (through overglorification of the trade, or the staging of tools and attributes amidst collective action).

\section{INDEX}

Keywords : guilds, fraternity, mutual assistance, initiatic group, image, lithography, cryptic teaching, work, trade, profession

Mots-clés : compagnonnage, fraternité, assistance mutuelle, tour de France, société initiatique, image, lithographie, enseignement crypté, métier

\section{AUTEUR}

\section{LAURENT BASTARD}

Laurent Bastard, né en 1955, est issu d'une famille de cinq générations de compagnons tanneurscorroyeurs du Devoir au XIXe et XXe siècle. Il est directeur du musée du Compagnonnage de Tours depuis 1993. Il a été commissaire-adjoint de l'exposition "Le Compagnonnage, chemin de l'excellence", au musée national des arts et traditions populaires à Paris (16 novembre 1995 - 6 mai 1996). Il a publié de nombreux articles dans des revues d'histoire et dans la collections des Fragments d'histoire du Compagnonnage éditée par le musée depuis 1999. Il est l'auteur de Travail et Honneur, les compagnons tailleurs de pierre en Avignon aux XVIIIe et XIXe siècles (en collaboration avec Jean-Michel Mathonière), Dieulefit, La Nef de Salomon (1995) ; Compagnons au fil de la Loire, Paris, Editions Jean-Cyrille Godefroy (2000) ; Chefs-d'oeuvre de compagnons ; Ed. De Borée (2008) ; Images des compagnons du Tour de France, Paris, Editions Jean-Cyrille Godefroy (2010). 